\title{
Enabling Optimal Power Generation of Flow Cell Arrays in 3D MPSoCs with On-Chip Switched Capacitor Converters
}

\author{
Halima Najibi*, Jorge Hunter $\$$, Alexandre Levisse*, Marina Zapater*†, Miroslav Vasic $\ddagger$, David Atienza* \\ *Embedded Systems Laboratory (ESL), EPFL, Switzerland \\ $\dagger$ REDS Institute, University of Applied Sciences Western Switzerland (HEIG-VD, HES-SO), Switzerland \\ \#Centro de Electrónica Industrial, UPM, Spain
}

\begin{abstract}
Flow cell arrays (FCAs) provide efficient on-chip liquid cooling and electrochemical power generation capabilities in three-dimensional multi-processor systems-on-chip (3D MPSoCs). When connected to power delivery networks (PDNs) of chips, the current flowing between FCA electrodes partially supplies logic gates and compensates over $20 \% V_{d d}$ drop in highperformance 3D systems. However, operation voltages of CMOS technologies are generally higher than the voltage corresponding to the maximal FCA power generation. Hence, directly connecting FCAs to 3D MPSoC power grids results in sub-optimal performance. In this paper, we design an on-chip direct current to direct current (DC-DC) converter to improve FCA power generation in high-performance 3D MPSoCs. We use switched capacitor (SC) technology and explore different design space parameters to achieve minimal area requirement and maximal power extraction. The proposed converter enables a stable and optimal voltage between FCA electrodes. Furthermore, it allows us to dynamically control FCA connectivity to 3D PDNs, and switch off power extraction during chip inactivity. We show that regulated FCAs generate up to $123 \%$ higher power with respect to the case they are directly connected to 3D PDNs. In addition, connecting multiple flow cells to a single optimized converter reduces area requirement down to $1.26 \%$, while maintaining IRdrop below $5 \%$. Finally, we show that activity-based dynamic FCA switching extends by over $1.8 \times$ and $4.5 \times$ electrolytes lifetime for a processor duty-cycle of $50 \%$ and $20 \%$, respectively.

Index Terms-3D MPSoCs, FCA technology, on-chip power generation, 3D power delivery networks, DC-DC converter.
\end{abstract}

\section{INTRODUCTION}

Three-dimensional multi-processor systems-on-chip (3D MPSoCs) enable energy-efficient high density computing and provide ultra-wide communication bandwidth requirements for next-generation applications [1]. However, they are generally challenged by rising heat dissipation difficulty with the number of stacked dies, due to higher power consumption per surface unit and low thermal conductivity of bonding layers. Furthermore, 3D integration increases power delivery complexity in multi-processor architectures due to resistive losses in through-silicon-vias (TSVs) and metal wires, as well as $3 \mathrm{D}$ routing congestion constraints [2]. In this context, flow cell array (FCA) technology, first introduced in [3], promises to address 3D thermal and power challenges. FCAs consist of micro-fluidic channels etched in the silicon substrate of $3 \mathrm{D}$ MPSoC dies. They provide combined on-chip liquid cooling and power generation capabilities due to heat-accelerated electrolyte reactions. When connected to 3D MPSoC power networks, FCA-generated current partially powers logic gates and reduces voltage drop across metal lines, therefore limiting timing violations and system performance degradation [4].

As FCAs connect to 3D MPSoC power delivery networks (PDNs), their generated power depends on the voltage between flow cell electrodes. In particular, peak power generation is achieved with operation voltages between $0.55 \mathrm{~V}$ to $0.62 \mathrm{~V}$ for vanadium redox-based FCAs, with fluid temperatures of $30^{\circ} \mathrm{C}$ and $60^{\circ} \mathrm{C}$, respectively [4] [5] [6]. However, $V_{d d}$ values in state-of-the-art high performance systems are generally between $0.75 \mathrm{~V}$ and $1.2 \mathrm{~V}$ [7]. Therefore, directly connecting FCA electrodes to 3D PDNs leads to sub-optimal power generation performance and prevents full exploitation of FCA benefits. In this context, we design in this paper an onchip switched capacitor (SC) voltage regulator to serve as an interface between FCAs and 3D MPSoC PDNs. The proposed SC converter enables optimal operation of FCAs, by providing a stable voltage that leads to maximal power generation. Furthermore, it allows to disconnect FCAs in case of chip inactivity, hence preventing excess input power that is dissipated by delivery grid resistance and chip leakage. Our contributions can be summarized in the following:

- We design a direct current to direct current (DC-DC) converter to supply a stable voltage to FCAs in 3D MPSoCs, leading to optimal on-chip power generation performance. We use SC technology and explore different design-space parameters to achieve high power density and low area requirement [8] [9].

- We show that using the proposed voltage regulator, FCAs generate up to $123 \%$ higher power compared to the case where they are directly connected to the PDN of a highperformance 3D MPSoC, operating at $1.1 \mathrm{~V} \quad V_{d d}$ and achieving a fluid temperature up to $41{ }^{\circ} \mathrm{C}$.

- We show that optimizing DC-DC converters to connect multiple FCA cells allows us to reduce the overall additional area requirement to less than $1.26 \%$, keeping IRdrop across the chip under the $5 \% V_{d d}$, which is a typical constraint of high-performance IC designs [4].

- We show that controlling FCA connectivity to 3D MPSoCs power networks using SC converters prevents electrolyte reactions during chip inactivity. Hence dynamically switching on and off FCA power generation extends by up to $1.8 \times$ FCA reservoir lifetime for a $3 \mathrm{D}$ MPSoC duty-cycle of $50 \%$, and by over $4.5 \times$ for $20 \%$ duty-cycle. 


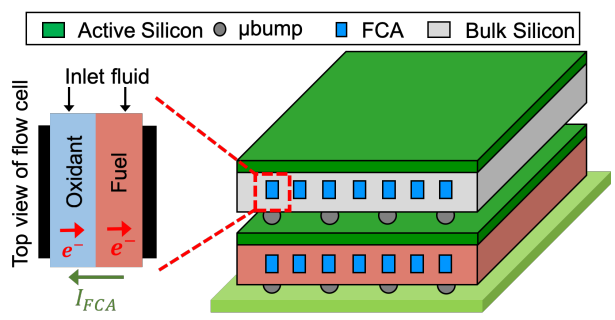

Fig. 1: 3D MPSoC with integrated FCAs

\section{BACKGROUND AND RELATED WORK}

\section{A. 3D MPSoC Design with Flow Cell Arrays}

3D MPSoCs are getting the attention of IC design engineers due to promising advantages in terms of computing density, heterogeneity, and communication bandwidth [1]. However, TSV-based 3D integration presents additional challenges related to thermal and power management. Power density increases with the number of stacked dies, generating heat that becomes difficult to dissipate due to the low thermal conductivity of silicon and other bonding materials [10]. In addition, power delivery is challenging as higher currents traverse power delivery TSVs and metal lines, causing a voltage drop that affects system performance. In this regard, the FCA technology addresses 3D MPSoC power and thermal challenges, by providing combined on-chip liquid cooling and electrochemical power generation [3] [4]. FCAs use microchannels etched in the silicon substrate of 3D MPSoC dies, as shown in Figure 1. The channels are filled with an electrolytic liquid flow that adsorbs heat generated by the switching activity of chips. Furthermore, high channel temperatures increase the rate of the electrochemical reactions, which generates an electrical current that can be supplied to logic gates. Hence, FCAs effectively transform heat into available generated power in high-density and high-performance 3D MPSoCs [3].

FCA power generation depends on both the voltage between electrodes and liquid temperature. For vanadium-based redox flows used in this work, peak power generation is achieved around $0.6 \mathrm{~V}$, for different liquid temperatures, as shown in Figure 2. The authors in [4] propose a design and analysis methodology for 3D MPSoCs with integrated FCAs, using a fine-grained electro-thermal simulator [6]. For a lossless use of on-chip generated power, they directly connect FCA electrodes to nearby power delivery metal lines in the back-end-of-line (BEOL) of dies. They show that FCA-current can reduce the voltage drop across the power grid of a high-performance processor by $20 \%$, hence improving voltage delivery network efficiency, at no extra cost in TSVs or grid density. In the same way, FCAs allow to relax power delivery system requirements of 3D MPSoCs with specific voltage constraints [4]. Although directly connecting FCAs to 3D MPSoC PDNs shows important improvements in power efficiency, their power generation capabilities are limited when operating at $V_{d d}$ of highperformance systems, typically around $1 V$. Hence, voltage regulation is critical to ensure optimal performance of FCAs, and full exploitation of their potential.

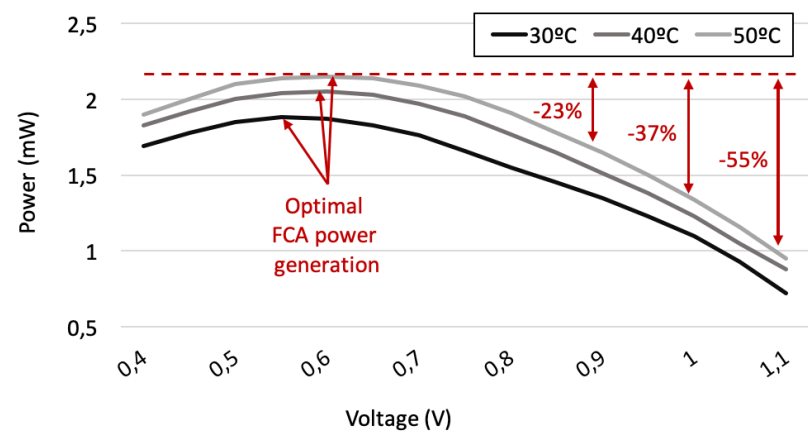

Fig. 2: FCA-generated power ( $50 \mu m$ width, $100 \mu m$ height and $100 \mu \mathrm{m}$ cell length) with respect to voltage and temperature

\section{B. Voltage Regulator Design for Efficient IC Power Delivery}

Voltage Regulators (VRs) are used for power management of modern 3D-ICs, providing voltage levels that are different than the standard printed circuit board (PCB) supply. Integrated VRs also enable fast voltage scaling to improve core performance while maintaining acceptable power consumption and voltage-drop levels [11]. In addition, VRs allow to decouple power supply from logic circuits and keep constant voltage levels in case of supply noise and transient load changes. The most common types of VRs used in ICs to step up voltage are switching regulators, which function by temporarily storing charge in magnetic or electric fields and then discharging it at a different voltage level. Two main types of switching regulators exist: inductor-based [12] and capacitor-based [13] [14].

Inductor-based switching regulators, used in IC power delivery networks, generally consist of buck-boost or buck-boostderived topologies [15] [16]. They can achieve high power conversion efficiency rates and are easy to control. However, on-chip integration of inductors remains an important challenge of inductor-based VRs. Particularly, the low quality of air-core spiral inductors makes them unsuitable for ICs [15], and microfabricated inductors, although promising, are not well developed yet [16]. A successful implementation of an inductor-based VR is found in 4th generation Intel Core SoCs [12]. This integrated VR design achieves a maximum conversion efficiency of $90 \%$ and output power of $108 \mathrm{~W}$, but has a very large area requirement of $175 \mathrm{~mm}^{2}$, of which $160 \mathrm{~mm}^{2}$ are occupied by inductors alone.

Capacitor-based converters, known as SC converters, consist exclusively of capacitors and transistor switches. In comparison to inductor-based VRs, SC converters are easier to integrate and require significantly lower chip area. Common SC converter designs achieve efficiencies up to $80 \%$ [13] [14], sizing around $0.3 \mathrm{~mm}^{2}$ for an output power of $4.2 \mathrm{~mW}$. Furthermore, designs including new technologies such as deep trench capacitors [17] can achieve $85 \%$ efficiency with $30 \times$ lower area requirement. Due to the significantly lower cost in chip area, we focus in this work on capacitor-based technology to design a converter that meets voltage and power efficiency requirements of 3D MPSoCs with integrated FCAs. 


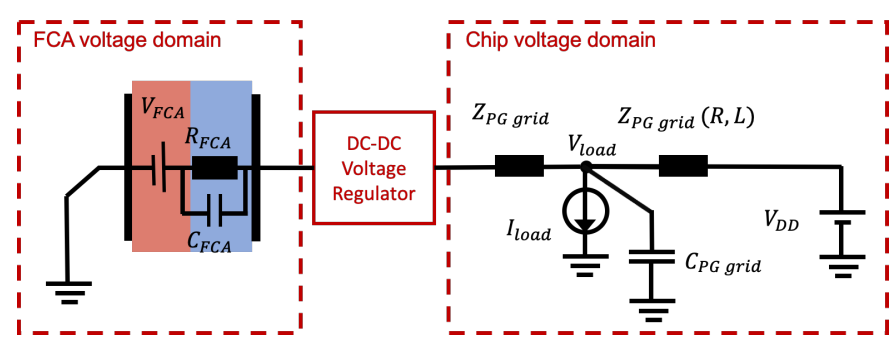

Fig. 3: FCA connectivity to the 3D MPSoC power delivery grid via integrated DC-DC voltage regulator
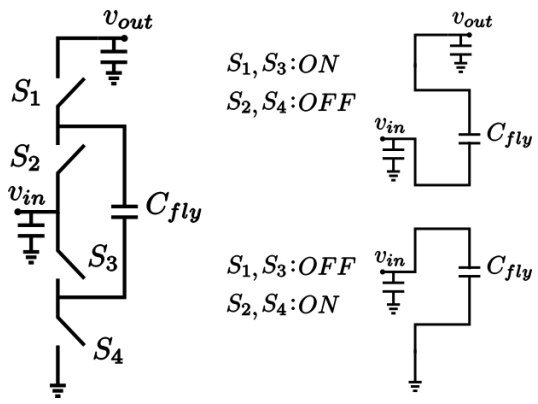

Fig. 4: 1:2 SC Converter Circuit

\section{INTEGRATED SC DC-DC CONVERTER DESIGN FOR FCA Voltage REgulation}

This section describes the design methodology of a 1:2 SC converter to provide optimal FCA operation voltage. Thus, it connects FCA cells to 3D MPSoC power grids, each with their respectful voltage domain, as shown in Figure 3. It is primarily designed for minimal area, while aiming for the highest possible output power.

\section{A. SC Converter State-Space Model}

The typical 1:2 SC converter consists of four transistors and a flying capacitor $C_{f l y}$, as shown in Figure 4. This topology can be simplified to the equivalent converter circuit model in Figure 5. It comprises of two resistors $R_{v}$ and $R_{i}$, modeling conduction and switching losses, respectively, and a capacitor $C_{e q}$ to account for first-order circuit dynamics. For a target output voltage $V_{\text {out }}=V_{d d}$ and FCA operation voltage $v_{i n}=v_{F C A}$, the resistances $R_{v}$ and $R_{i}$ are determined as $R_{v}=\frac{V C R * v_{\text {in }}-v_{\text {out }}}{i_{\text {out }}}$ and $R_{i}=\frac{v_{\text {out }}}{\frac{i_{\text {in }}}{V C R}-i_{\text {out }}}$, where the Voltage Conversion Ratio (VCR) corresponds to the particular $\mathrm{SC}$ topology. The capacitive component $C_{e q}$ is calculated by analyzing circuit equations in the Laplace domain [18]. Thus, characterizing a given SC converter design requires to determine the electrical parameters of its circuit. To do so, a matrix-based methodology is developed [19], and improved taking into account the significant effect of on-chip bottom plate capacitance [20]. In this paper we use this generalized methodology to the specific use-case of FCAs, taking into account their electrical characteristics. Although the design and modeling steps are applicable to any SC converter topology, we apply it exclusively to a 1:2 VCR converter.

For any given SC converter, the following matrices and vectors are defined, where $\mathbf{n}$ is the number of capacitors and

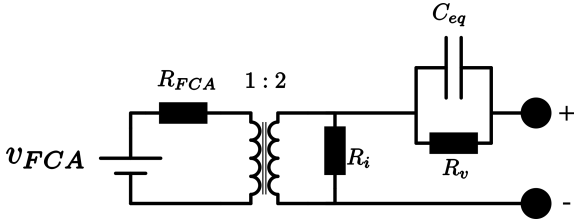

Fig. 5: 1:2 SC Equivalent Converter Model

$\mathbf{i}$ and $\mathbf{v}$ are the current and voltage across each capacitor, respectively:

$\mathbf{C}=\left[\begin{array}{cccc}C_{1} & 0 & \cdots & 0 \\ 0 & C_{2} & \cdots & 0 \\ \vdots & \vdots & \ddots & 0 \\ 0 & \cdots & \cdots & C_{n}\end{array}\right] \mathbf{i}=\left[\begin{array}{c}i_{C_{1}} \\ \vdots \\ i_{C_{n}}\end{array}\right] \mathbf{v}=\left[\begin{array}{c}v_{C_{1}} \\ \vdots \\ v_{C_{n}}\end{array}\right] \mathbf{U}=\left[\begin{array}{c}v_{\text {in }} \\ v_{\text {out }}\end{array}\right]$

Applying Kirchhoff's laws to the circuit, $2 n$ independent equations are derived in the form of Equation 2.

$$
\mathbf{A i}+\mathbf{B v}+\mathbf{D U}=\mathbf{0}
$$

From which $\mathbf{i}$ can be isolated as follows:

$$
\mathbf{i}=-\mathbf{A}^{-1} \mathbf{B v}-\mathbf{A}^{-1} \mathbf{D U}
$$

Considering the capacitor's fundamental equation $i=C \dot{v}$, $\dot{\mathbf{v}}$ is expressed as:

$$
\dot{\mathbf{v}}=-\mathbf{C}^{-1} \mathbf{A}^{-1} \mathbf{B v}-\mathbf{C}^{-1} \mathbf{A}^{-1} \mathbf{D U}
$$

Which is a state-space equation of the form:

$$
\dot{\mathbf{x}}=\mathbf{A x}+\mathbf{B u}
$$

This process is repeated for each switching phase $i$ of the $\mathrm{SC}$ converter. By solving the state-space equation 5 of each phase, we obtain a solution in the form:

$$
\mathbf{v}_{\mathbf{i}}(\mathbf{t})=\boldsymbol{\Phi}_{\mathbf{i}}(\mathbf{t}) \mathbf{v}(\mathbf{0})+\Gamma_{\mathbf{i}}(\mathbf{t}) \mathbf{U}
$$

Assuming that in steady-state $\mathbf{v}((\mathbf{k}+\mathbf{1}) \mathbf{T})=\mathbf{v}(\mathbf{k} \mathbf{T})$, we calculate $\mathbf{v}(\mathbf{0})$, the equilibrium value of the voltage at the beginning of each cycle. Hence, we calculate $\Delta \mathbf{v}$ and derive the charge across each capacitor over $\mathrm{T}$. This procedure allows to approximate the expected converter output, and build the equivalent circuit model from Figure 5.

Additionally, with few modifications to the proposed procedure, first-order converter dynamics are calculated and incorporated into the circuit model. To reduce our analysis to the first-order dynamics, we compute the dominant eigenvalue from the state transition matrix $\left(\Phi_{i}\right)$ which represents most of the dynamics of the system. The discrete model of the system can then be described as follows:

$$
\mathbf{y}[\mathbf{k}+\mathbf{1}]=\lambda_{\max } \mathbf{y}[\mathbf{k}]+\left(1-\lambda_{\max }\right)\left(g_{1} v_{\text {in }}+g_{2} v_{\text {load }}\right)
$$

This equation can be readily transformed into the Laplace domain from which the output impedance $Z_{\text {out }}$ is derived. It is represented as a parallel RC branch in our model, taking into account $R_{v}$ and $C_{e q}$. 


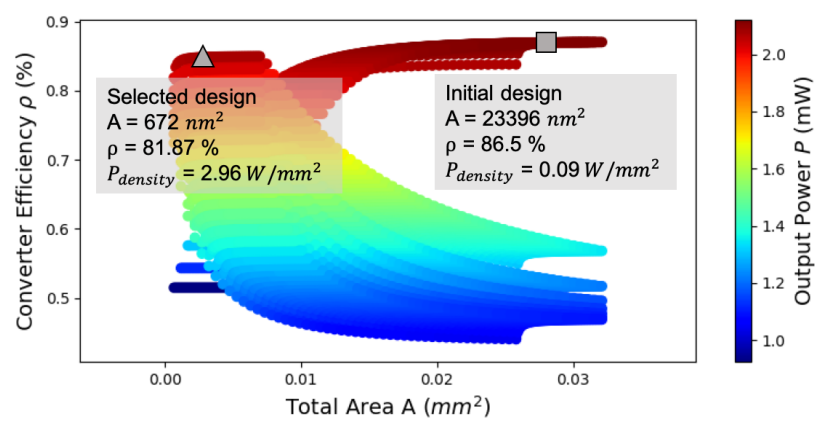

Fig. 6: Evaluated DC-DC converter design points

\section{B. Design Space Parameters}

To design an SC converter, typical design parameters are transistor and capacitor sizing, and switching frequency:

- Transistor and capacitor sizing determines the SC converter circuit resistance and capacitance, which are important components responsible for conduction and switching losses, respectively.

- Switching frequency directly affects power loss in the circuit. In general, $P_{l o s s} \propto \frac{1}{k \times f_{c l k}}$, where $k$ depends on the converter topology. However, in the case of highperformance ICs, this dependence does not hold for higher frequencies, as additional losses occur due to parasitic components [14].

The effects of each design parameter on switching and conduction losses are correlated. Hence, for a given design we apply the methodology in Section III-A to calculate the converter performance parameters for different configurations. To build the state-space model, we use transistor and capacitor models from a 32nm CMOS technology [17].

\section{Converter Design Exploration}

The implemented state-space model allows us to calculate converter performance for a given target output voltage. Indeed, modern processors typically operate at different $V_{d d}$ values depending on load and performance. Besides, design points that are optimized for a given output voltage are not necessarily optimal at slightly varying output voltages. In this context, we develop an algorithm that cycles through a wide range of combinations of design parameters, with over 10 million evaluated design points. Since the converter is expected to output several voltage levels, the performance results are weighted according to the target load profile to obtain averaged performance metrics.

Figure 6 shows different evaluated SC converter design points with respect to their total area requirement, their voltage conversion efficiency, and output power. This figure indicates a clear trade-off between efficiency, output power, and area. In particular, output power scales slower than area. In this work, we select the converter design represented by a star in Figure 6 for FCA voltage regulation. This design has the lowest area requirement and the highest output power density. Alternatively, depending on the design constraints, other configurations can be selected with higher efficiency or output power.

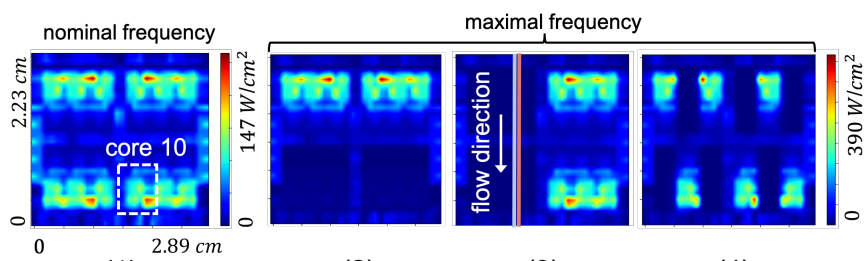

(1)

(2)

(3)

(4)

Fig. 7: Real POWER8 processor powermaps

\section{EXPERIMENTAL SETUP}

To evaluate FCA performance using the proposed SC converter, we design a two-layer 3D MPSoC composed of a multicore computing layer and a memory layer. The memory layer contains four $2^{\text {nd }}$ generation HBM memories with 4 DRAM layers. Each HBM has a base die size of $71 \mathrm{~mm}^{2}$, and consumes $15 W$ [21]. We base the architecture and power profile of the processing layer on the 12-core IBM POWER8 processor [7], and consider its implementation in $32 \mathrm{~nm}$ CMOS technology (used to build the converter state-space model). The processor die size is $649 \mathrm{~mm}^{2}$ and its power consumption is $190 \mathrm{~W}$, with a highly non-uniform powermap. Then, FCAs of $50 \mu \mathrm{m}$ width and $100 \mu \mathrm{m}$ height are etched in the silicon substrate of both dies with a pitch of $50 \mu \mathrm{m}$, as shown in Figure 1. Each $200 \mu m$-long flow cell section is connected to a single $\mathrm{SC}$ converter, which is in turn connected to the dies power grids. Furthermore, TSVs are arranged in groups delivering power to independent subgrids. Their diameter and pitch are both fixed at $5 \mu \mathrm{m}$. Finally, $V_{d d}$ is fixed at $1.1 \mathrm{~V}$, corresponding to the maximum processor performance.

During full utilization, the processor in the proposed 3D MPSoC has a higher power consumption than the memory. Therefore, we focus on it to evaluate FCA performance, while the memory layer activity contributes to the overall temperature increase. Thus, we use the four real measured POWER8 powermaps, shown in Figure 7, to evaluate the voltage regulator and FCA power generation efficiency in different load scenarios. All powermaps contain multiple high power density regions mainly concentrated in the computing cores. In powermap (1) from Figure 7, all cores are operating at nominal frequency. In powermaps (2), (3) and (4) six cores operate at maximal frequency and achieve peak power density, while the others are idle (e.g. awaiting data from the memory).

We perform a fine-grained simulation of the 3D MPSoC and assess the thermal and PDN performance. To do so, we use cell dimensions of $200 \times 100 \mu m^{2}$ and $50 \times 50 \mu m^{2}$ for the thermal and electrical simulation, respectively. In this way, we evaluate the FCA power generation, converter efficiency, and voltage map. We build a compact flow cell model corresponding to the voltage-power dependency in Figure 2, and a converter circuit model (Figure 5) to perform dynamic SPICE simulations. The cores are switched between 3 different activity levels: idle, nominal, and maximal frequency operation (according to powermaps in Figure 7). As dies have individual PDNs, the total number of FCAs and converters in the 3D MPSoC scales linearly with the number of layers, while their performance depends on the load and voltage level of each die independently. 


\section{RESUlTS AND Discussion}

\section{A. Optimized FCA Power Generation}

We measure the FCA power generation and DC-DC converter efficiency when cores switch between different activity levels. Particularly, we select core 10 to demonstrate FCA power generation improvement using the proposed converter, as it shows different load and liquid temperature levels (Figure 7). Hence, Figure 8 shows HSPICE transient analysis results of the processor power grid. All three plots present FCAgenerated current when directly connected to the power grid operating at $V_{d d}$, FCA-generated current when operating at the optimal voltage enabled by voltage regulation, and output current of the DC-DC converter, when used. Three nodes are selected from the POWER8 core, which correspond to different power densities: maximum (Figure 8a), medium (Figure 8b) and low (Figure 8c).

Our results show that FCAs generate for all the cases over four times higher current when operating at their optimal voltage, supplied via the DC-DC converter, with respect to the case they operate at $V_{d d}$ of the chip. Thus, FCAs generate up to $123 \%$ additional power due to voltage regulation. Furthermore, peak FCA power generation is achieved in case the load corresponds to powermap (3) for all three evaluated nodes, due to higher liquid temperature inside the channels, as it passes two high power density cores. Our results also indicate that DC-DC converter output power remains over $90 \%$ higher than FCA-generated power when no voltage regulation is used, corresponding to over $82 \%$ conversion efficiency. Using the DC-DC converter allows us to boost the power generation capabilities of individual FCA cells in 3D MPSoCs regardless of load levels of their corresponding chip area, but requires a large number of converters, costing area and design complexity. In this context, we explore in section V-B different configurations in terms of the number of FCA cells per converter.

\section{B. DC-DC Converter Area Optimization}

To reduce the number and area requirements of DC-DC converters in 3D MPSoC dies, we explore different design options in terms of the number of FCA cells connected to each converter. To minimize FCA power dissipation in the metal wires to the converter, we group cells in squares so that each $N \times N$ connect to one converter. For each configuration, the input power density of the converter is proportional to the number of connected FCA cells. Therefore, the DC-DC converter is optimized accordingly, for maximal conversion efficiency, following the methodology described in Section III-C. Consequently, by taking into account the resistance of wires connecting FCA cells to converters, we perform a DC voltage analysis of the processor. To do so, we select powermap (3) in Figure 7, which contains the largest concentration of power hotspots, and therefore induces the most critical IR-drop.

Figure 9 presents the maximal IR-drop at the processor when connecting 1 to 36 FCA cells to each DC-DC voltage regulator. As multiple FCA cells connect to a single converter, their output currents traverse longer wires to reach all the loads they supply. Particularly, $10 \mathrm{mV}$ additional IR-drop occurs

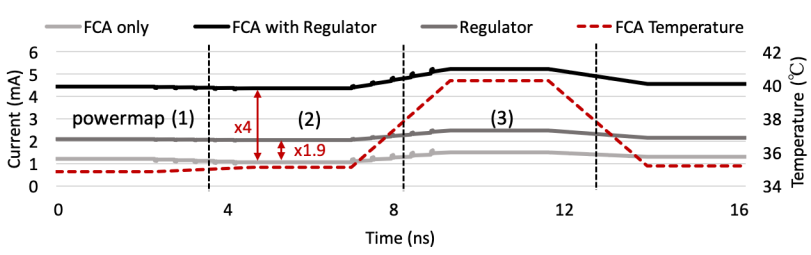

(a) Maximum power consumption node

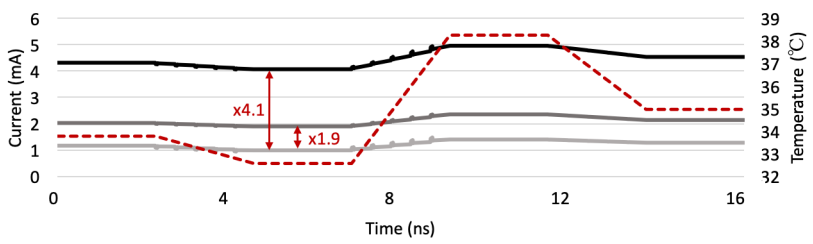

(b) Medium power consumption node

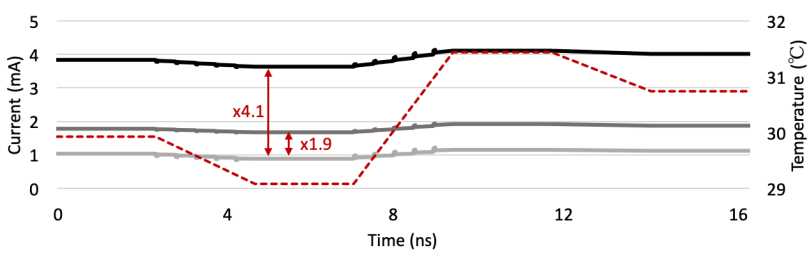

(c) Low power consumption node

Fig. 8: FCA and DC-DC regulator output currents when switching between the different powermaps

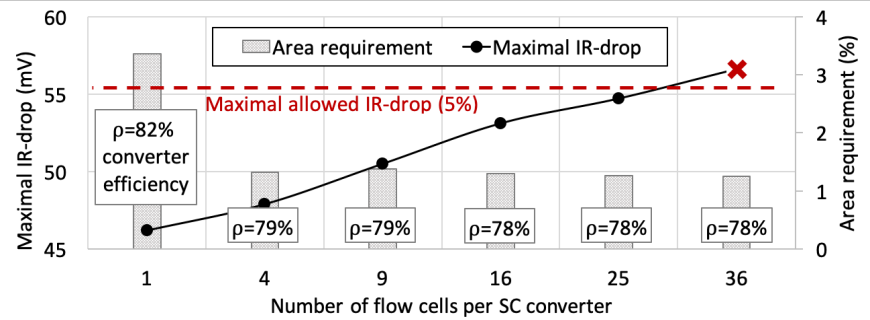

Fig. 9: Maximal IR-drop, converter area requirement and efficiency for different number of FCA cells per converter, at maximal processor power consumption with $V_{d d}=1.1 \mathrm{~V}$

when connecting up to 36 flow cells to one converter. Figure 9 also shows the total area percentage requirement of optimized converters for each configuration, and their efficiency. This figure indicates that connecting 25 FCA cells to each converter saves over $2.5 \%$ of total chip area, while maintaining the maximal IR-drop value under $5 \%$ (typical voltage constraint in high-performance ICs [4]). In addition to area savings, decreasing the number of converters by $25 \times$ reduces design and routing complexity in $3 \mathrm{D}$ MPSoCs with integrated FCAs.

\section{Dynamic FCA Switching}

FCA power generation occurs due to electrochemical reactions between electrolytes inside channels. Continuous extraction of power gradually decreases the concentration of reactants, as they are being consumed. Electro-thermal simulations demonstrate that, when liquid flows through FCA channels, up to $1.1 \%$ and $0.9 \%$ of its electrolytes react, in full load and idle chip scenarios respectively. Furthermore, electrolytes crossover that occurs along membrane-less flow cells causes 


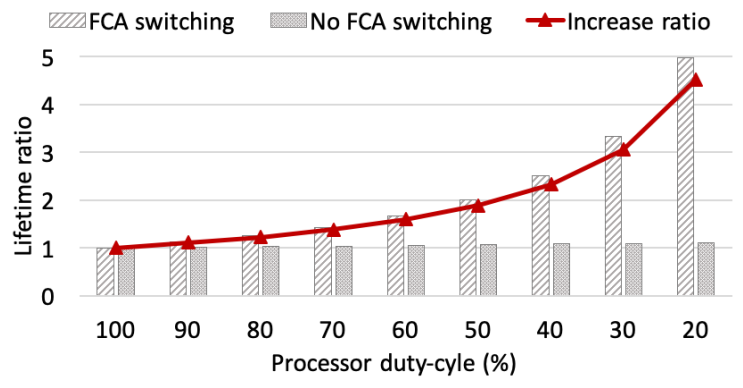

Fig. 10: FCA reservoir lifetime with and without FCA power switching, for different processor duty-cycle values, normalized to the lifetime at $100 \%$ processor duty-cycle

contamination of the oxidant and fuel, and decreases their concentration regardless of reaction rate. Generally, for high-speed flow cells, up to $40 \mathrm{~mA} / \mathrm{cm}^{2}$ current is generated by electrolyte crossover in an open-circuit scenario, corresponding to $0.5 \%$ of generated current in optimal FCA power generation conditions [22]. As a result, using DC-DC converters, we can dynamically disconnect FCAs from 3D MPSoC PDNs, and hence prevent excess power extraction when the chip is idle or in low-power operation. In fact, when disconnected, only FCAs cooling capabilities are used, and fuel concentration degradation is limited to cross-contamination effects.

Based on the previous analysis, Figure 10 illustrates the lifetime of a fuel reservoir in different processor duty-cycle levels, with and without dynamically switching FCA power extraction (on and off), as well as the ratio between the two scenarios (in red). When connected to the processor power grid, FCAs operate at their optimal voltage, enabled by DC-DC conversion. FCA lifetime is normalized to the value corresponding to continuous power extraction and chip operation (i.e $100 \%$ duty-cycle). Our results show that, with $50 \%$ duty-cycle of the processor, dynamic switching of FCA power generation extends by over $1.8 \times$ the lifetime of a fuel reservoir, as only crossover occurs and a large amount of electrolytes are not used up, when no extra power is needed. When the chip is only active $20 \%$ of time, dynamic switching extends by over $4.5 \times$ the FCA reservoir lifetime. In general, using DC-DC converters clearly improves FCA durability.

\section{CONCLUSION}

In this paper, we have evaluated the power generation performance of FCAs using high efficiency and low area SC converters. The proposed converters serve as an interface to connect FCA electrodes to 3D MPSoC PDNs. They allow operating flow cells at the voltage value that leads to optimal power generation capability, with $V_{d d}$ supply of highperformance multi-core platforms. Thus, we have explored different design space parameters to meet the area and output power requirements of the DC-DC converter. Consequently, we have shown that voltage regulators enable up to $123 \%$ higher on-chip power generation, with respect to directly connecting FCAs to 3D MPSoC power grids. Furthermore, connecting multiple FCA cell segments to a single optimized converter allows us to limit the cost in total chip area to less than $1.26 \%$. Finally, the proposed converters enable controlling FCA connectivity to 3D MPSoC power grids, based on chip activity. Hence, our results have indicated that dynamically switching on and off the FCA power extraction increases between $1.8 \times$ and $4.5 \times$ the FCA reservoir lifetime for a processor duty-cycle of $50 \%$ and $20 \%$, respectively.

\section{ACKNOWLEDGMENTS}

This work has been partially supported by the ERC Consolidator Grant COMPUSAPIEN (GA No. 725657) and the EC H2020 WiPLASH (GA No. 863337).

\section{REFERENCES}

[1] F. Clermidy et al. 3D Embedded Multi-Core: Some Perspectives. Design, Automation and Test in Europe Conference (DATE), 2011.

[2] P. Sivakumar et al. Optimization of thermal aware multilevel routing for 3D IC. Analog Integrated Circuits and Signal Processing, 2019.

[3] A. Andreev et al. PowerCool: Simulation of Cooling and Powering of 3D MPSoCs with Integrated Flow Cell Arrays. IEEE Transactions on Computers (TC), 2018.

[4] H. Najibi et al. A Design Framework for Thermal-Aware Power Delivery Network in 3D MPSoCs with Integrated Flow Cell Arrays. International Symposium on Low Power Electronics and Design (ISLPED), 2019.

[5] A. Andreev et al. Design Optimization of 3D Multi-Processor Systemon-Chip with Integrated Flow Cell Arrays. ISLPED, 2018.

[6] A. Sridhar et al. PowerCool: Simulation of integrated microfluidic power generation in bright silicon MPSoCs. International Conference on Computer Aided Design (ICCAD), 2014.

[7] E. Fluhr et al. POWER8: a 12 core server-class processor in $22 \mathrm{~nm}$ SOI with 7.6Tb/s off-chip bandwidth. International Solid-State Circuits Conference (ISSCC), 2014.

[8] A. Paul et al. Deep Trench Capacitor Based Step-Up and Step-Down DC/DC Converters in 32nm SOI with Opportunistic Current Borrowing and Fast DVFS Capabilities. IEEE Asian Solid-State Circuits Conference (A-SSCC), 2013.

[9] S. Banzhaf et al. Post-Trench Processing of Silicon Deep Trench Capacitors for Power Electronic Applications. International Symposium on Power Semiconductor Devices and ICs (ISPSD), 2016.

[10] E. Wong et al. 3D Floorplanning with Thermal Vias. DATE, 2006.

[11] P. Vivet et al. A 220GOPS 96-Core Processor with 6 Chiplets 3D-Stacked on an Active Interposer Offering $0.6 \mathrm{~ns} / \mathrm{mm}$ Latency, $3 \mathrm{~Tb} / \mathrm{s} / \mathrm{mm}^{2}$ Inter-Chiplet Interconnects and $156 \mathrm{~mW} / \mathrm{mm}^{2} @ 82 \%$ Peak-Efficiency DC-DC Converters. ISSCC, 2020.

[12] E. A. Burton et al. FIVR - Fully Integrated Voltage Regulators on 4th Generation Intel ${ }^{\circledR}$ Core ${ }^{\mathrm{TM}}$ SoCs. Proceedings of the IEEE Applied Power Electronics Conference and Exposition, 2014.

[13] T. V. Breussegem and M. Steyaert. A $82 \%$ efficiency $0.5 \%$ ripple 16phase fully integrated capacitive voltage doubler. IEEE Symposium on VLSI Circuits, 2009.

[14] D. Somasekhar et al. Multiphase $1 \mathrm{GHz}$ voltage doubler charge-pump in $32 \mathrm{~nm}$ logic process. IEEE Journal of Solid State Circuit (JSSC), 2010.

[15] T. M. Andersen. On-Chip Switched Capacitor Voltage Regulators for Granular Microprocessor Power Delivery. PhD thesis, ETH Zürich, 2015.

[16] H. W. Koertzen P.R. Murrow, C. Park and J. T. DiBene. Design and fabrication of on-chip coupled inductors integrated with magnetic material for voltage regulators. IEEE Transactions on Magnetics, 2011.

[17] T. M. Andersen et al. A feedforward controlled on-chip switchedcapacitor voltage regulator delivering $10 \mathrm{~W}$ in $32 \mathrm{~nm}$ SOI CMOS. ISSCC, 2015.

[18] L. Muller and J. W. Kimball. A Dynamic Model of Switched-Capacitor Power Converters. IEEE Transactions on Power Electronics (TPE), 2014.

[19] J. M. Henry and J. W. Kimball. Practical Performance Analysis of Complex Switched-Capacitor Converters. TPE, 2011.

[20] T. M. Andersen et al. Modeling and Pareto Optimization of On-Chip Switched Capacitor Converters. TPE, 2017.

[21] D. Lee et al. A 1.2 V 8Gb 8-Channel 128GB/s High-Bandwidth Memory (HBM) Stacked DRAM With Effective I/O Test Circuits. JSSC, 2015.

[22] A. S. Hollinger et al. Nanoporous separator and low fuel concentration to minimize crossover in directmethanol laminar flow fuel cells. Journal of Power Sources, 2010. 